\title{
Modelling of Echo Amplitude Fidel iy for Transducer Bandwidth and TFM Pixel Resolution
}

David Lines $^{1}$, Ehsan Mohseni ${ }^{1}$, Rastislav Zimermann ${ }^{1}$, Carmelo Mineo ${ }^{2}$, Charles MacLeod $^{1}$, Gareth Pierce ${ }^{1}$, Anthony Gachagan ${ }^{1}$

david.lines@strath.ac.uk

${ }^{1}$ Centre for Ultrasonic Engineering, University of Strathclyde, UK

2University of Palermo, Italy 


\section{Outline}

- Motivation

- Temporal Sampling rates

-1D Model for Parametric Analysis

- Rectification approaches

- Parametric Analysis Results

- Model extension for FMC+TFM

- TFM Parametric Analysis Results

- Conclusions and Future Work 


\section{Motivation 1 - Temporal Sampling}

- Temporal and Amplitude sampling needs to be sufficient to reconstruct the waveform so that echo amplitude and time-of-flight can be estimated to the required level of accuracy

- Temporal sampling has typically been done at around 20 samples per cycle (20x Fc)

- The large number of A-scans in each Full Matrix Capture (FMC) frame means transferring data at 20x Fc can be the limiting parameter for the frame acquisition rate

- Optimizing temporal sampling can remove this constraint 


\section{Motivation 2 - Spatial Sampling}

- Similar considerations about echo amplitude fidelity are required for B-scan images, which determine the minimum pixel pitch

- For Total Focusing Method (TFM) imaging, the ASME V standard requires the maximum amplitude measurement error to be $<2 \mathrm{~dB}$

- For smooth images the pixel pitch has typically been between 10 and 20 pixels per cycle

- The heavy processing load to generate each pixel in the TFM image is a strong incentive to reduce the sampling resolution as much as possible, so as to increase the image reconstruction rate

- This must not violate the $2 \mathrm{~dB}$ amplitude fidelity criterion in ASME V 


\section{Temporal Sampling Rates}

- Investigated by many: Edmund Whittaker (1915); Harry Nyquist (1928); Vladimir Kotelnikov (1933); Claude Shannon (1948), etc. This work resulted in the criterion:

- Temporal Sampling rate $>2 x$ maximum frequency in signal

- Shannon's modified case for band-limited signals (1949)

- Temporal Sampling rate $>2 x$ bandwidth of signal

- Practical systems don't have a hard cut-off frequency

- Fractional bandwidth is usually specified at the $-6 \mathrm{~dB}$ points

- Better to use -20dB cut-offs to minimise aliasing

- For Gaussian spectra, $-20 \mathrm{~dB}$ width is $\sim 1.85 \mathrm{x}$ the $-6 \mathrm{~dB}$ width

- $\rightarrow$ Temporal Sampling rate $\geq 4 x$ Fractional Bandwidth 


\section{D Model for Parametric Analysis}

- Model was developed using LabVIEW ${ }^{\circledR}$ (National Instruments)

- Test signal is Gaussian-windowed sine wave sampled at $1 \mathrm{GS} / \mathrm{s}$, with control over:

- Amplitude \& Phase of sine (RF) component

- Offset of Gaussian window

- Standard deviation of Gaussian window

- Noise can be added (but is not considered here)

- Reference (RF) for comparison uses typical instrument values:

- $50 \mathrm{MHz}$ sampling at $12 \mathrm{bits}$

- Echo amplitude measurement approaches are compared with the Test signal amplitude, over variations in:

- Test Signal Phase $\left(0\right.$ to $\left.360^{\circ}\right)$

- Gaussian window 0 ffset $\left(-180^{\circ}\right.$ to $\left.180^{\circ}\right)$

- Test Signal's -6dB Fractional Bandwidth (Gaussian window Standard Deviation) 


\section{Rectification Approaches}

- RF: full sample rate

- Max amplitude error when samples equi-spaced around peak

- Error depends on samples/cycle

- Full Wave Rectification

- ABS(RF)

- Same amplitude errors as RF

- Envelope (Hilbert)

- $\sqrt{R F^{2}+(\operatorname{Hilb}(R F))^{2}}$

- Amplitude error depends on bandwidth
Sample Rate $=50 \mathrm{MHz}$ at 12 bits Signal at $5 \mathrm{MHz}$ with $50 \%$ Fractional Bandwidth

Signal phase variation: $360^{\circ}$ in $2^{\circ}$ steps

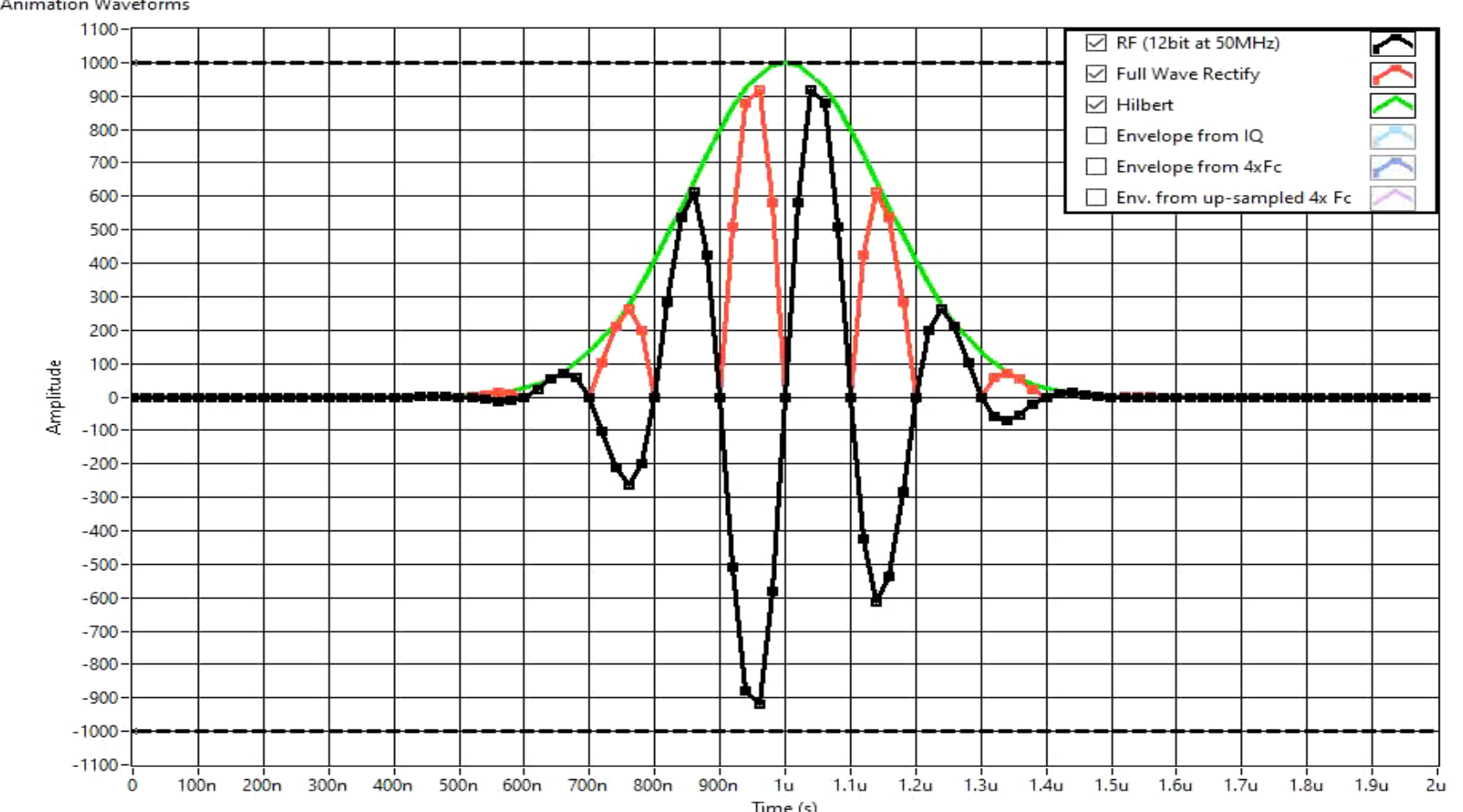




\section{Rectification Approaches}

- RF: full sample rate

- Quadrature Sampling

- Amplitude errors vary with signal phase

- Sample at $4 x$ Fcentre

- $\sqrt{4 F c^{2}+(H i l b(4 F c))^{2}}$

- Amplitude errors less affected by phase

- Up-sampled from 4x Fc

- $4 x F c$ up-sampled $(50 \mathrm{MHz})$ \& envelope as for Hilbert

- Errors similar to original
Sample Rate $=50 \mathrm{MHz}$ at 12bits Signal at $5 \mathrm{MHz}$ with $50 \%$ Fractional Bandwidth

Signal phase variation: $360^{\circ}$ in $2^{\circ}$ steps

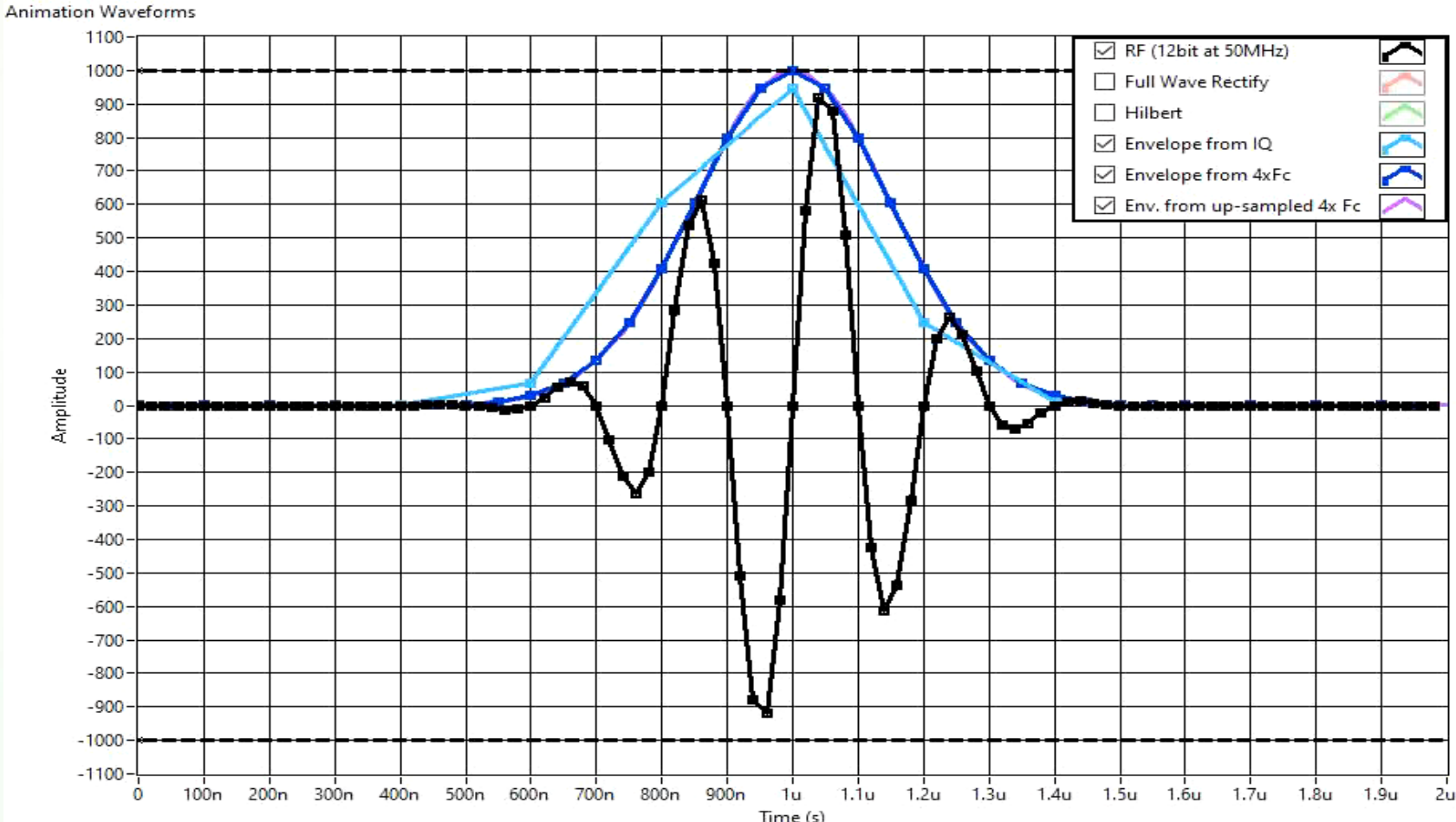




\section{Effect of Bandwidth on RF}

$\mathrm{RF}$ from $4 \mathrm{xFc}$ vs. Original as fractional bandwidth varies from $30 \%$ to $150 \%$

Offset $=45^{\circ}$, for worst case sampling around peak

- 4xFc samples

- Up-sampled from $4 x F c$

- Original RF waveform

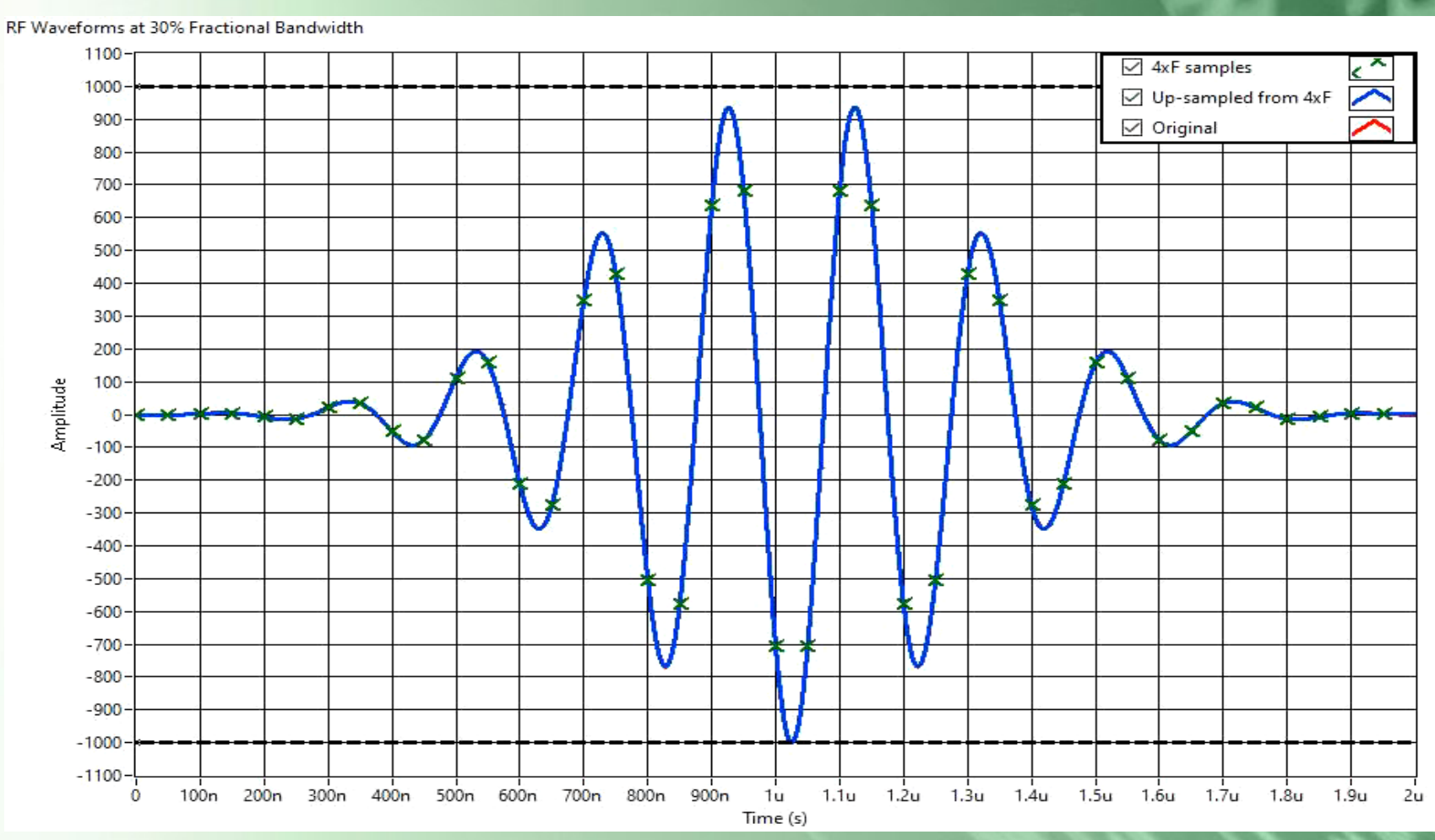




\section{Parametric Analysis Results}

Max Amplitude error vs. Fractional Bandwidth
Sample Rate $=50 \mathrm{MHz}$ at $12 \mathrm{bits}$ Signal at $5 \mathrm{MHz}$

Phase \& Offset varied in $5^{\circ}$ steps
Full Wave Rect. (50MHz)

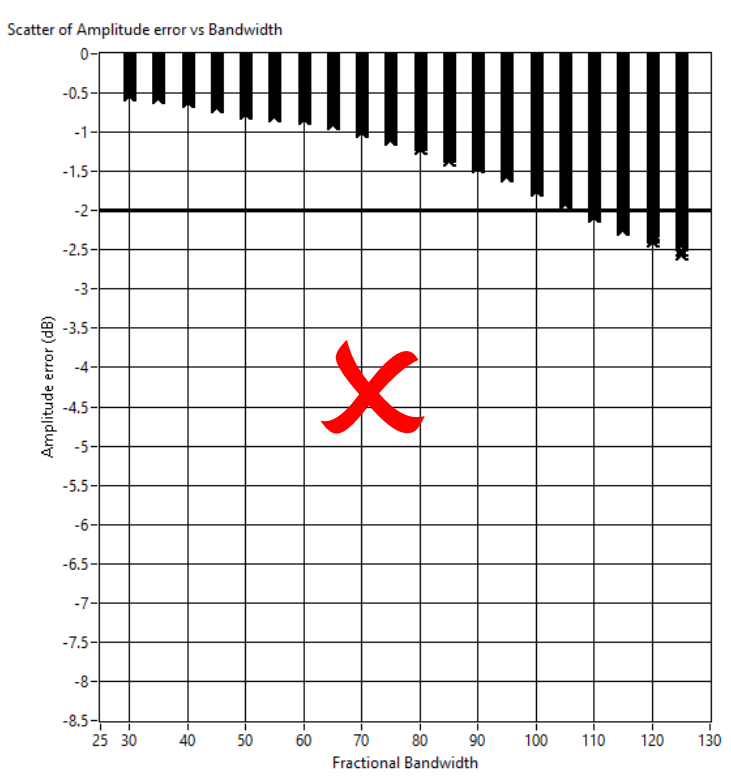

Envelope (Sampled 50MHz)

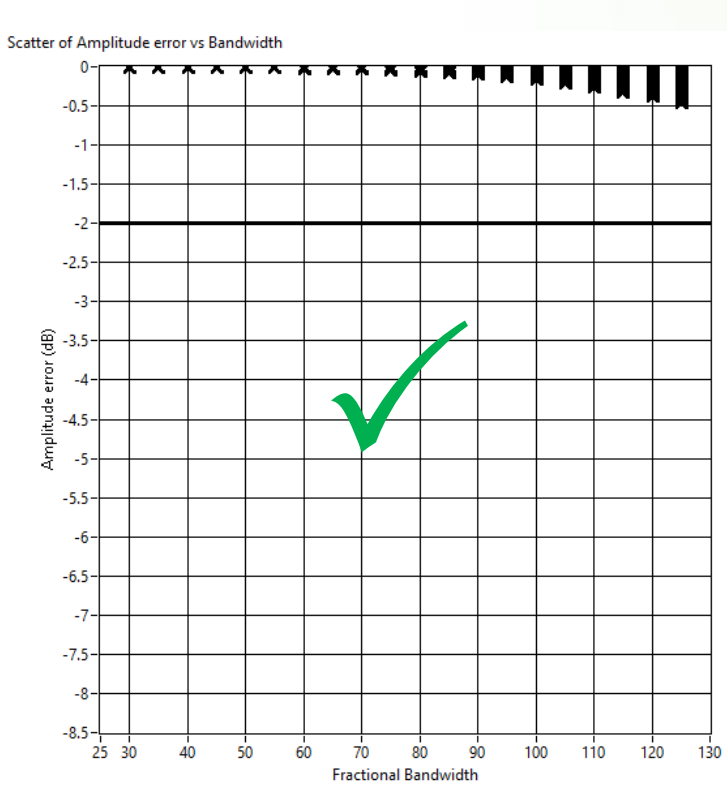

Envelope (Sampled IQ $\equiv 10 \mathrm{MSPS})$

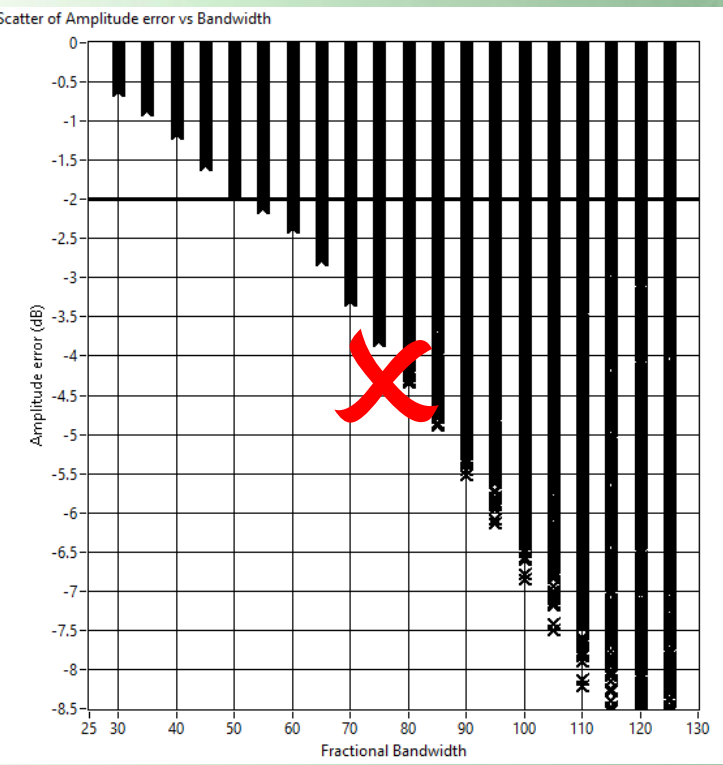

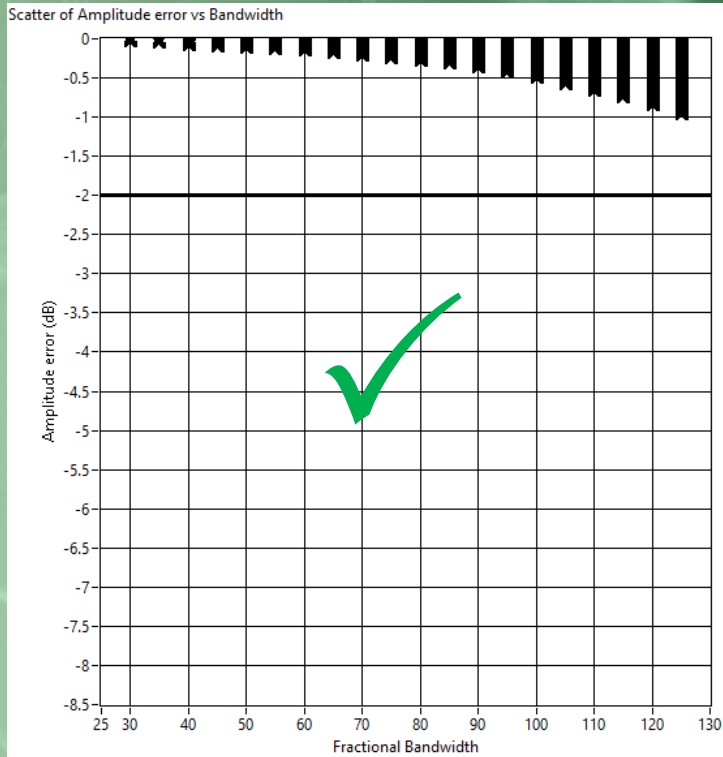




\section{D Parametric Analysis Summary}

- The Parametric Analysis has confirmed that the best echo amplitude estimate should be made by envelope detection

- Maximum error of $-0.173 \mathrm{~dB}$ at Fractional Bandwidth of $100 \%$

- The RF signal representation and envelope detection are best on the standard practice of over-sampling at 10x or 20x centre frequency.

- However, sampling at $4 x$ transducer centre frequency is confirmed to achieve the necessary accuracy even at high bandwidth transducers

- Maximum error of $-0.284 \mathrm{~dB}$ at Fractional Bandwidth of $100 \%$

- Since $4 x \mathrm{Fc}$ sampling is able to represent the waveform to the required accuracy, it will be acceptable to sample the FMC data at this rate 


\section{Model extension for FMC+TFM}

- 2D imaging model is an extension of the $1 \mathrm{D}$ model to analyse temporal sampling

- FMC data is created by simulating all the individual A-scans between each element pair for a point target

- It uses the same Gaussian-windowed sine wave, sampled at $1 \mathrm{GS} / \mathrm{s}$, as for the 1D model

- The round trip transit time, from transmit element to point reflector and back to receive element, is derived and used to interpolate the $1 \mathrm{GS} / \mathrm{s}$ into the A-scan

- An amplitude weighting, corresponding to element directivity, is possible

- It is not used here so as to simplify the calculation of the amplitude error

- Each A-scan is then sampled at the specified rates before inserting into the FMC matrix

- The TFM RF image around the position of the point target is created using the standard complex summation of contributions from all A-scans for each pixel

- Echo amplitude measurement approaches are compared with the Test signal amplitude, for specified pixel resolution (as pixels/wavelength), over variations in:

- Gaussian window 0 ffset $\left(-180^{\circ}\right.$ to $\left.180^{\circ}\right)$

- Reflector Angle

- Reflector Range

- Test Signal's -6dB Fractional Bandwidth (Gaussian window Standard Deviation) 


\section{Clarification of Wavelength $(\lambda)$ Terminology}

For pulse-echo, in the time taken for $1 \mathrm{RF}$ cycle, the location of the echo in the image moves by $\Lambda / 2$

To avoid any confusion, the spatial pitch should always be defined in terms of the number of pixels/cycle rather than pixels/wavelength

\section{RF TFM image}

$4 \mathrm{~mm} \times 4 \mathrm{~mm}$ TFM image of $45 \mathrm{~mm}$ range $0^{\circ}$ target at $20 \mathrm{pix} / \mathrm{cycle}=40 \mathrm{pix} / \Lambda$

- $f=5 \mathrm{MHz}$ at $50 \%$ bandwidth

- Velocity $(v)=5890 \mathrm{~m} / \mathrm{s}$

- $\lambda=\mathrm{v} / \mathrm{f}=1.18 \mathrm{~mm}$

64 element $0.5 \mathrm{~mm}$ pitch array

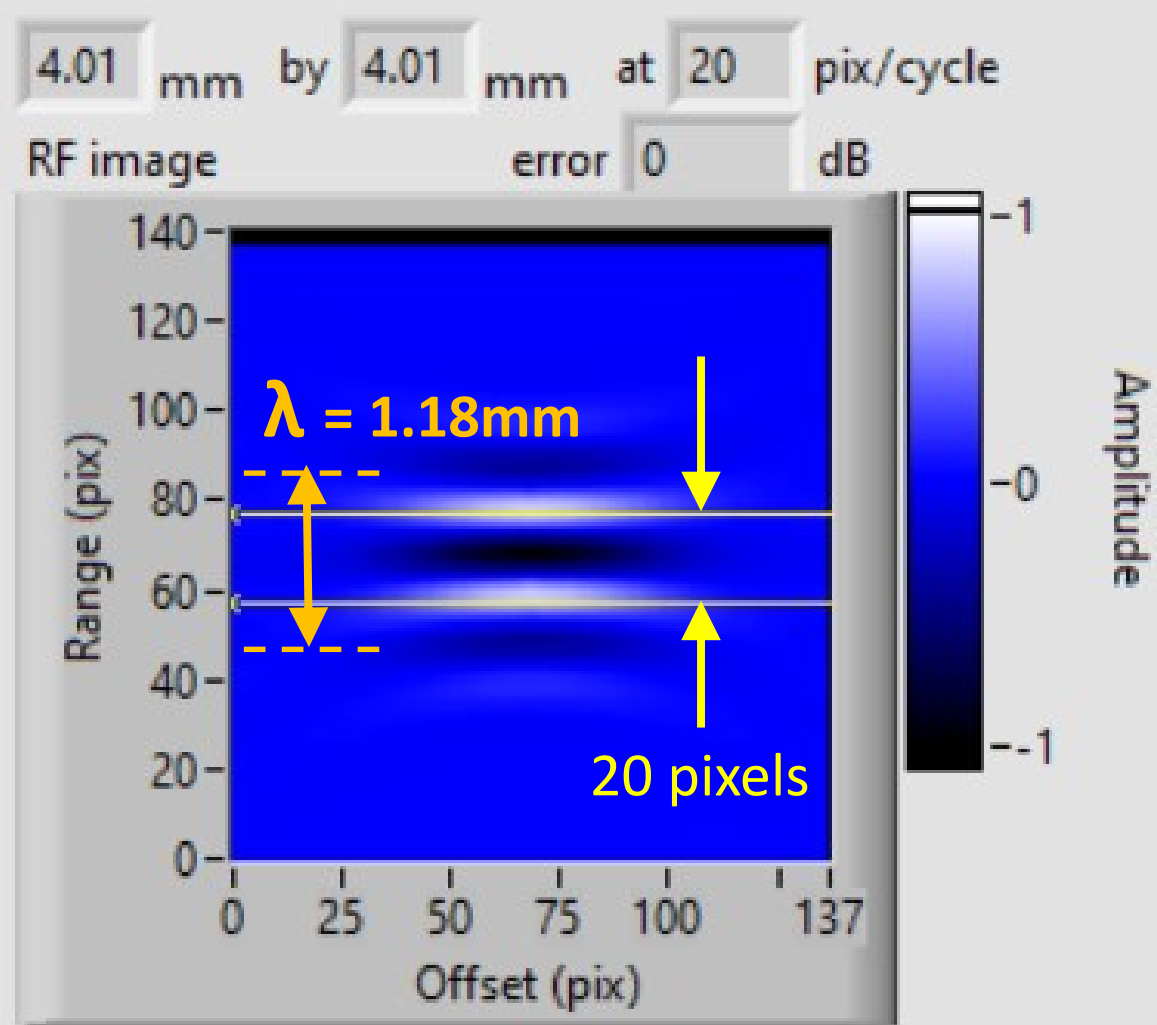




\section{Rationale for spatial sampling}

TFM images \& amplitude errors for $75 \%$ bandwidth on $45 \mathrm{~mm}$ range $50^{\circ}$ target at 20 and 4 pixels/cycle

- Axial resolution is typically better than lateral resolution

- Column sampling through a $0^{\circ}$ target is equivalent to the A-scan sampling already analysed so 4 pixels/cycle should give the same accurate rendition of the waveform

- 2D spatial up-sampling of the TFM RF image, before envelope detection, could give improved visual rendition without the processing time overhead of TFM generation at this resolution
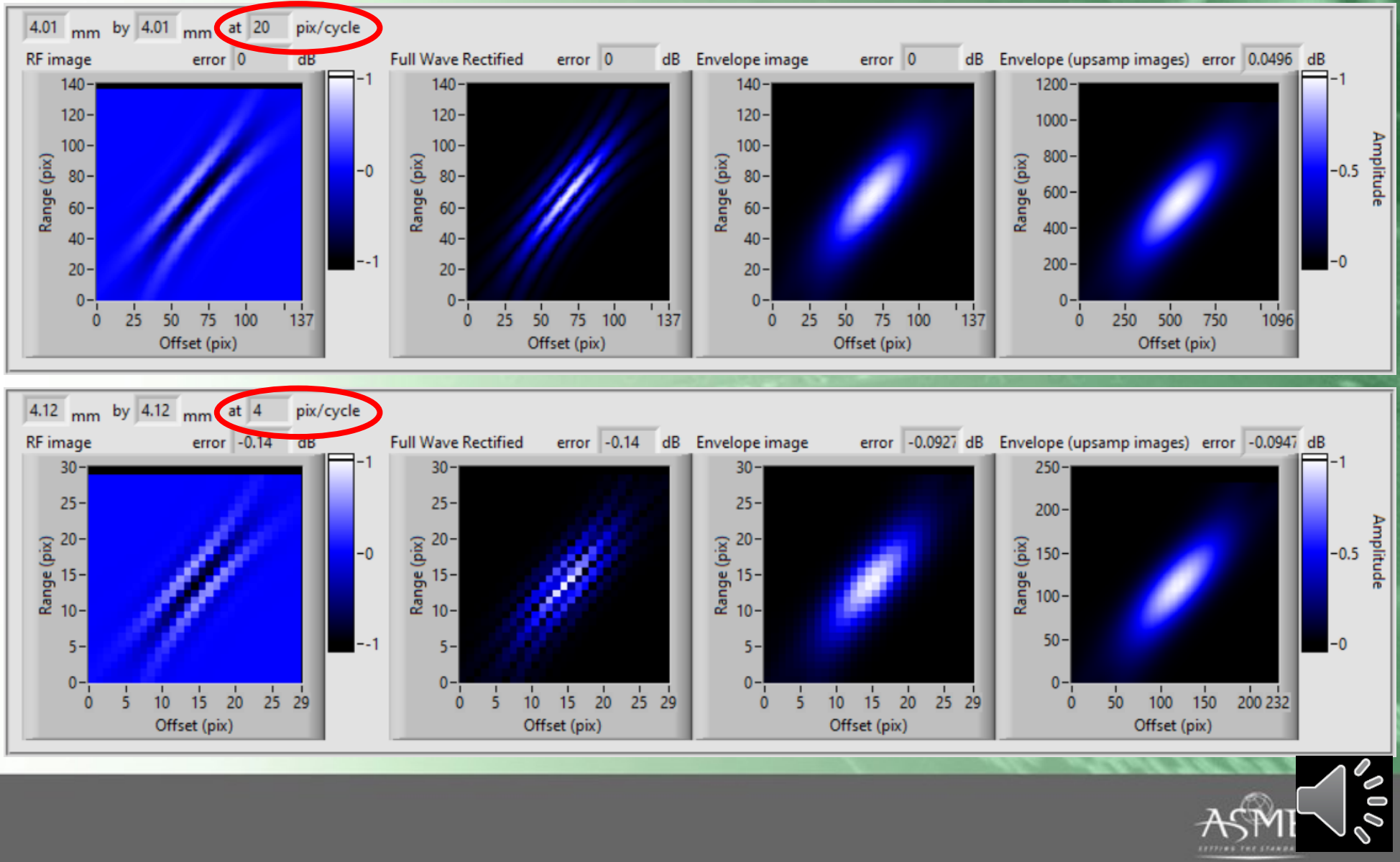


\section{Coarser spatial sampling}

TFM images \& amplitude errors for $75 \%$ bandwidth on $45 \mathrm{~mm}$ range $50^{\circ}$ target at 3 and 2 pixels/cycle

- ASME V criterion requires better than $2 \mathrm{~dB}$ echo amplitude fidelity

- Coarser sampling can offer higher throughput rates whilst achieving amplitude fidelity

- Error for Full Wave Rectification is $2.74 \mathrm{~dB}$ but still only $-0.341 \mathrm{~dB}$ for Envelope

- Up-sampling errors are similar but offer little benefit in improved rendition at coarser than 4 pixels/cycle
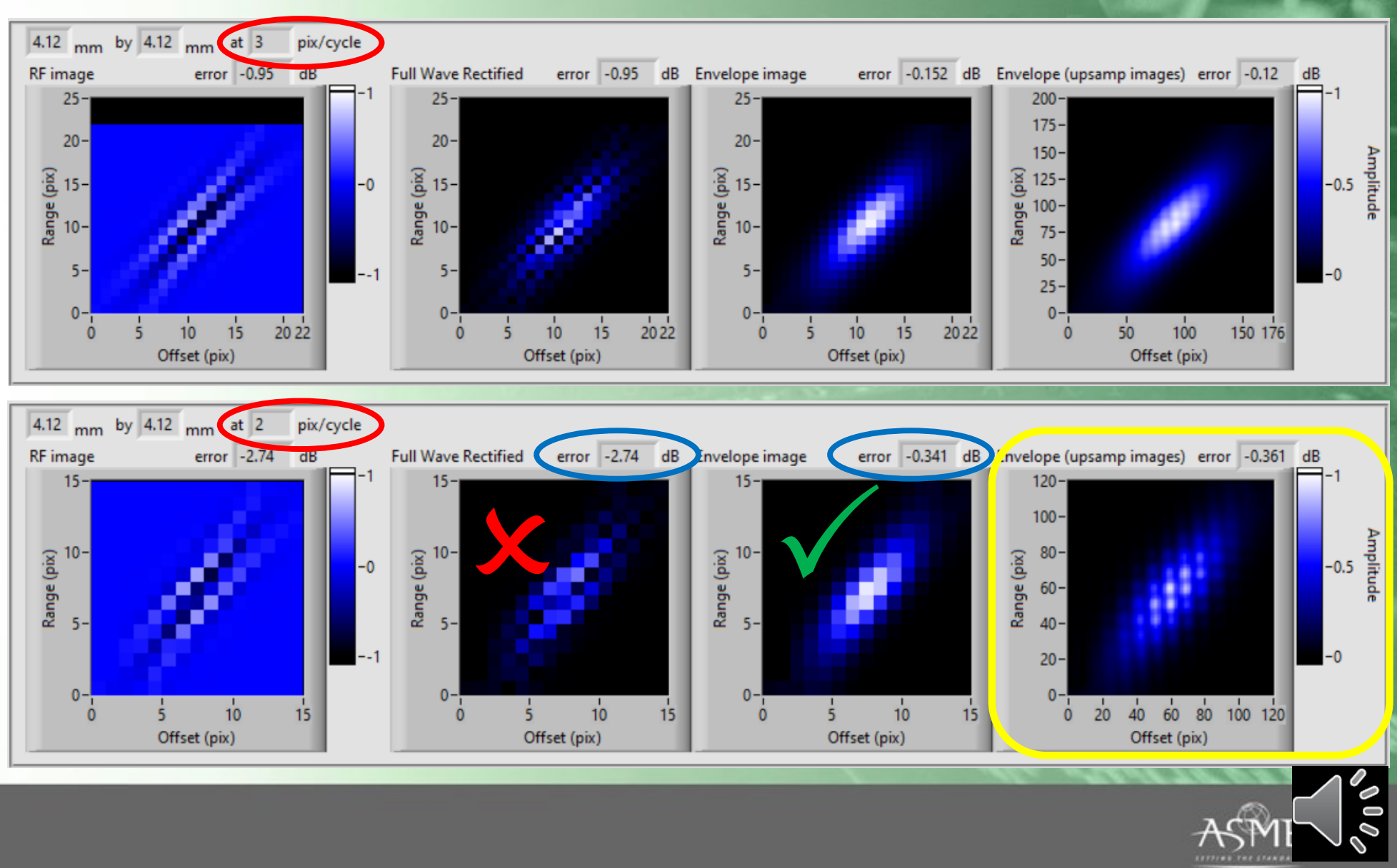


\section{Parametric analysis: Error Amplitude vs. Processing}

\section{Common parameters}

64 element $0.5 \mathrm{~mm}$ pitch contact array

Velocity $=5890 \mathrm{~m} / \mathrm{s}$

FMC data sampled at 50MHz 12bit

$7 \mathrm{~mm} \times 7 \mathrm{~mm}$ TFM reconstruction

Varying parameters

Bandwidths: $50 \%, 75 \%$ \& $100 \%$

Target Ranges: $30,45 \& 60 \mathrm{~mm}$

Target Angles: $0^{\circ}$ to $80^{\circ}$ in $5^{\circ}$ steps

Echo phases: $-180^{\circ}$ to $180^{\circ}$ in $5^{\circ}$ steps
Processing Modes:

0 : RF display

1 : Full Wave Rectification

2 : Envelope (at ADC rate)

3 : Envelope on spatially up-sampled (8x) images

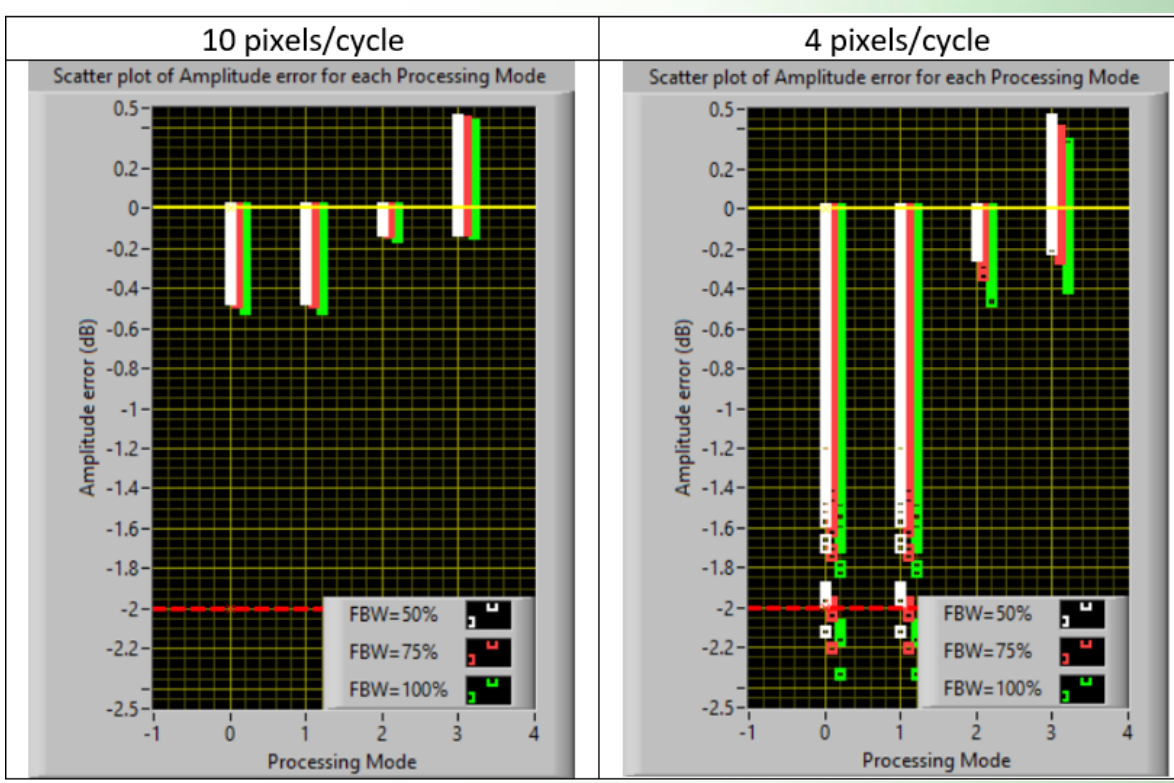

2 pixels/cycle

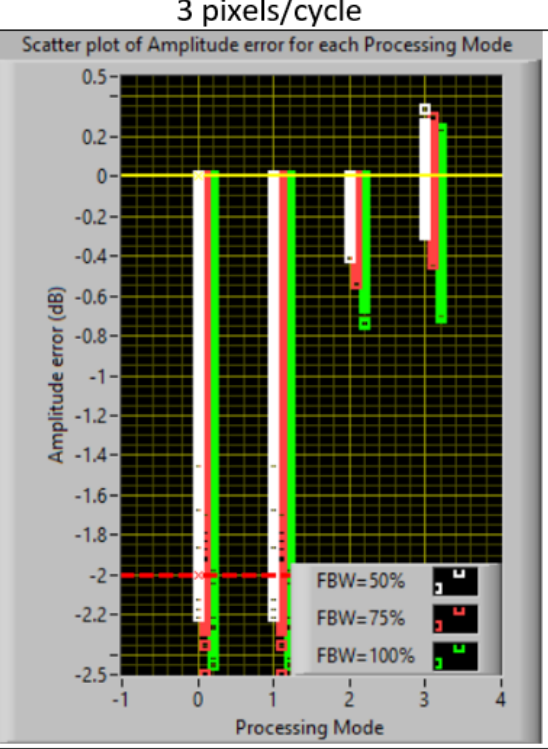

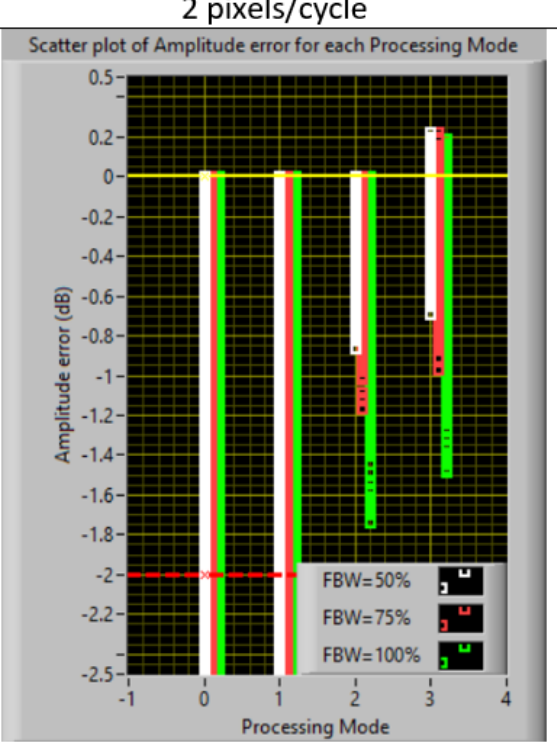

The Ame
$A S M E^{*}$ 


\section{Parametric analysis: Error Amplitude vs. Angle \& Ranġẹ}

Common parameters

64 element $0.5 \mathrm{~mm}$ pitch contact array

Velocity $=5890 \mathrm{~m} / \mathrm{s}$

FMC data sampled at $50 \mathrm{MHz} 12 \mathrm{bit}$

$7 \mathrm{~mm} \times 7 \mathrm{~mm}$ TFM reconstruction

2 pixels/cycle

Varying parameters

Bandwidths: $50 \%, 75 \%$ \& $100 \%$

Target Ranges: $30,45 \& 60 \mathrm{~mm}$

Target Angles: $0^{\circ}$ to $80^{\circ}$ in $5^{\circ}$ steps

Echo phases: $-180^{\circ}$ to $180^{\circ}$ in $5^{\circ}$ steps

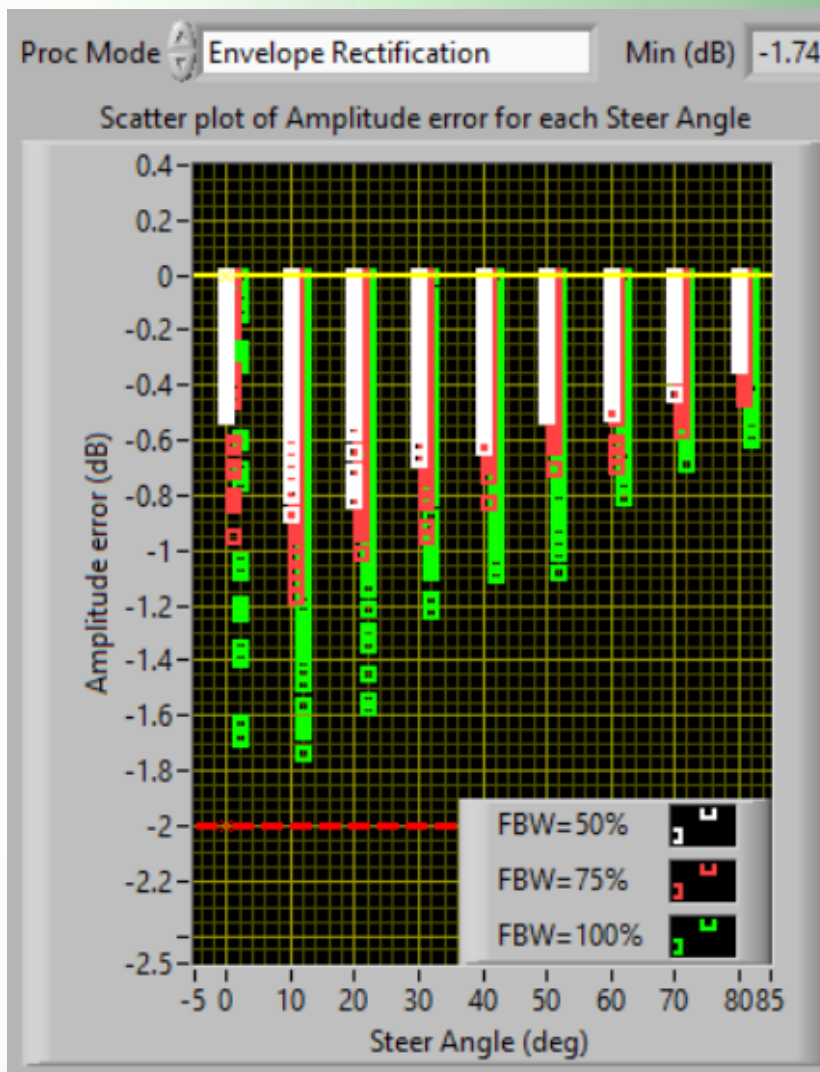

Steer Angle (deg)
Scatter plot of Amplitude error for each Range

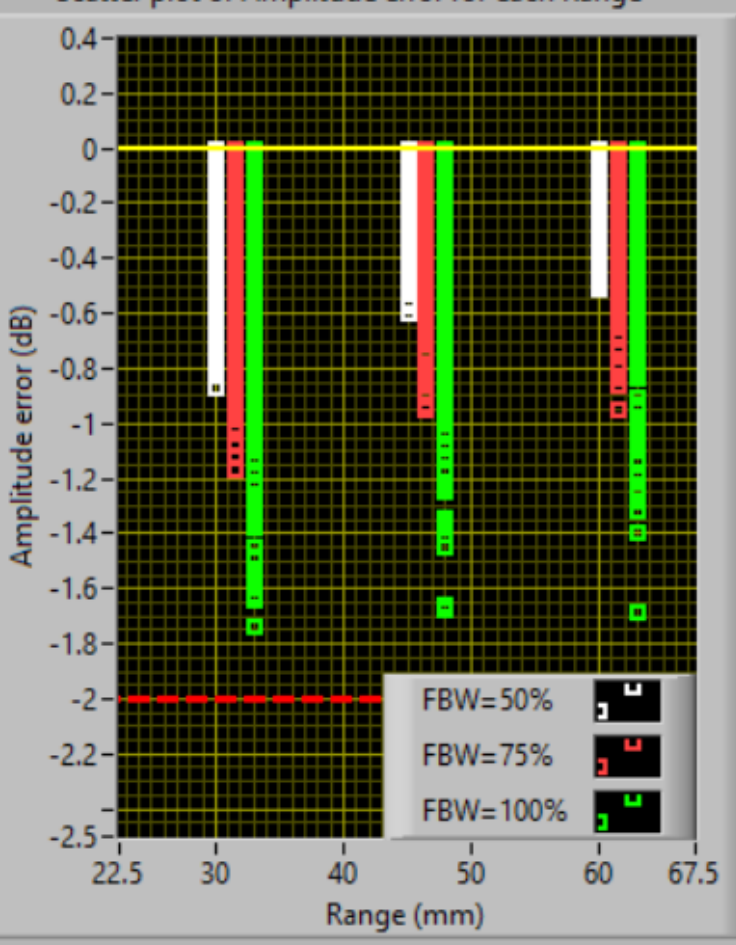




\section{Conclusions \& Future Work}

- Confirmed that temporal sampling of $4 x$ Centre Frequency is accurate for bandwidths up to $100 \%$

- Parametric analysis confirmed TFM spatial sampling of 4 pixels/cycle is accurate for same bandwidth range

- Coarser spatial sampling is possible whilst still achieving ASME V criterion (<2dB amplitude error)

- Coarse pixel sampling allows fast automated scanning with amplitude thresholding on TFM images

- If the threshold is exceeded, a finer pitch reconstruction can be done on the same FMC data for detailed review

Future Work

- Confirm that temporal sampling at $4 \mathrm{x}$ bandwidth is still valid for coded excitations 